Théologiques

Théologiques

\title{
La théologie dans une Église en déclin
}

\section{Normand Provencher}

Volume 14, numéro 1-2, automne 2006

Les lieux de la théologie aujourd'hui

URI : https://id.erudit.org/iderudit/014318ar

DOI : https://doi.org/10.7202/014318ar

Aller au sommaire du numéro

\section{Éditeur(s)}

Faculté de théologie et de sciences des religions, Université de Montréal

\section{ISSN}

1188-7109 (imprimé)

1492-1413 (numérique)

Découvrir la revue

\section{Citer cet article}

Provencher, N. (2006). La théologie dans une Église en déclin. Théologiques, 14(1-2), 177-188. https://doi.org/10.7202/014318ar

\section{Résumé de l'article}

Dans le contexte présent de la déchristianisation, la théologie devient de plus en plus discrète et isolée, comme l'Église qui est son «lieu» vital. La théologie court le risque de s'égarer dans un lieu où le "religieux" devient l'objet d'une science se prétendant objective et neutre. Le moment n'est-il pas venu de faire de la théologie dans un contexte d'exil, sur une terre étrangère? Dans cette situation, elle a l'occasion, mieux la grâce, de sortir de ses sentiers sacrés et de s'aventurer sur des chemins nouveaux. Elle peut prendre un second souffle, à la condition de se concentrer sur l'essentiel du mystère chrétien et d'être l'écho d'une parole unique et venue d'ailleurs.
Tous droits réservés @ Faculté de théologie et de sciences des religions, Université de Montréal, 2006
Ce document est protégé par la loi sur le droit d'auteur. L’utilisation des services d'Érudit (y compris la reproduction) est assujettie à sa politique d'utilisation que vous pouvez consulter en ligne.

https://apropos.erudit.org/fr/usagers/politique-dutilisation/ 


\title{
La théologie dans une Église en déclin
}

\author{
Normand PROVENCHER \\ Faculté de théologie \\ Université Saint-Paul
}

Je ne sais pas ce que deviendra la religion demain, mais je crois fermement à l'urgence de cette théologie pudique et radicale. (Certeau 1987, 263)

Le titre de cet exposé permet facilement d'identifier son auteur qui a publié récemment un livre intitulé Trop tard? L'avenir de l'Église d'ici (2002). Dans certains milieux, il a la réputation d'être pessimiste, alarmiste et, même, plus sociologue que théologien. Toutefois, on n'ose pas remettre en question sa description réaliste de l'Église d'ici. La majorité des lecteurs s'y reconnaît. En regard du diagnostic qu'il pose sur l'avenir de l'Église, les désaccords se font plus nombreux et parfois leur ton est agressif. En considérant lucidement ce qui se passe dans l'Église d'ici, nous ne pouvons plus prétendre qu'il ne s'agit que d'une crise passagère ou encore d'une mauvaise passe et qu'elle saura, une fois de plus, s'en sortir. Nous sommes les témoins, pour ne pas dire les acteurs, de la fin d'une Église qui a tant marqué notre pays. Est-elle donc engagée dans la phase finale de son existence ? La réponse à cette question m'est devenue claire : «Oui, c'est la fin d'une réalisation d'Église ». Ce n'est certes pas la fin de l'Église, encore moins du christianisme et de l'Évangile, mais la fin de cette Église, liée intimement à notre histoire, avec ses institutions, son clergé, son autorité.

Maurice Bellet constate la fin d'une forme de christianisme en Occident: "Quelque chose meurt: et nous ne savons pas jusqu'où cette mort descend en nous ". À ce constat, il ajoute aussitôt une note à la fois de réalisme et d'espérance: "Quelque chose s'annonce, et nous ne savons pas ce que ce sera. Mais c'est comme si nous étions sur la ligne de départ, 
à l'orée d'une nouvelle humanité. Pour le pire ? Pour le meilleur ? Nous ne savons pas; mais c'est largement entre nos mains. » $(2001,17)$ Dans une Église en déclin, pour ne pas dire en phase terminale, que devient alors la théologie? L'Église n'est-elle pas son lieu approprié et vital de production et de diffusion?

Louky Bersianik, une écrivaine de chez nous, affirme qu'il est plus difficile de voir le visible que l'invisible. En effet, rien n'est plus dérangeant que les faits; il n'est rien de plus difficile à détecter et à accepter que la vérité des faits. Il est évident que l’Église d'ici est affrontée à un déclin rapide et sans précédent. Ce déclin ne se ramène pas seulement au nombre décroissant des pratiquants, des prêtres et des religieuses, mais au fait que l'Église ne fonctionne plus «comme une institution fondatrice de sens fidèle à l'Évangile ". Dans nos sociétés désormais plurielles et de moins en moins chrétiennes, l'Église n'est plus l'autorité normative et inspiratrice. Sa parole est une parole parmi d'autres, et souvent non la plus crédible. Et cela, non seulement dans le domaine de l'éthique sexuelle, mais même dans des domaines qui normalement lui reviendraient, comme celui de la Bible, des origines du christianisme, de la spiritualité et même de Dieu. Étant engagé dans un débat avec Albert Jacquard qui a publié un livre intitulé Dieu ? ${ }^{1}$ (2003) et qui fut un succès étonnant de librairie, je suis arrivé à cette question: «Dieu serait-il en train d'échapper aux Églises ? " Michel de Certeau parlait déjà vers les années 1970 d'un «effondrement du corps ecclésial » et il n'hésitait pas à comparer l'Église à " une étoile dont la lumière se diffuse encore après sa disparition» $(1987,275$, note 6$)$. C'est dans ce contexte ecclésial et culturel qu'il nous faut situer la théologie. Il est étonnant que les théologiens et les théologiennes n'abordent pas, du moins sur la place publique, la question de l'avenir de leur discipline dans le contexte de la déchristianisation et du déclin de l'Église en Occident ${ }^{2}$.

\section{Théologie et vie de l'Église}

Un regard sur l'histoire nous permet de constater que le développement de la pensée chrétienne est lié étroitement à la vitalité et à la maturité de l’Église

1. À la suite d'un débat avec Albert Jacquard au Salon du livre de Québec, j’ai publié Dieu! Réponse à Albert Jacquard (2003).

2. Voir Bousquet et al. 2002 où, dans un livre de 885 pages et comprenant 64 contributions sur le rôle des théologiens, la question que pose à la théologie la situation de déclin de l'Église n'est pas abordée directement. Par contre, cette question est abordée avec beaucoup de lucidité par C. Duquoc (2002). 
(voir Lafont 2003). Pensons aux IV et $V^{e}$ siècles, l'ère de la rencontre de la foi chrétienne avec la pensée grecque; au XIII e siècle, l'ère des "sommes " de théologie et de la naissance des universités où la théologie était reine; au milieu du $\mathrm{XX}^{\mathrm{e}}$ siècle avec le concile Vatican II qui a encouragé le retour aux sources et le dialogue avec la société moderne. Ces moments féconds de la théologie ont jailli du dynamisme des chrétiens et chrétiennes de ces époques, à l'aise dans leur foi et dans leur société, même si les autorités de l'institution demeuraient discrètes et parfois inquiètes.

L'Église constitue le véritable lieu de la théologie, si nous acceptons pour programme la formule de saint Anselme au XI" siècle: "La foi cherchant à se rendre intelligible ». Il existe une complicité entre la foi et la raison. Or, l'Église n'est-elle pas le témoin authentique de la foi ? C'est dans la communauté confessante que le théologien la reçoit et qu'il la vit en communion avec les autres, notamment avec ceux et celles que l'Évangile appelle «les tout-petits» (Mt 11,25). En conséquence, parler du lieu concret de la théologie, c'est évoquer le lieu où s'insèrent les théologiens et les théologiennes. Ils font finalement la théologie du lieu qu'ils occupent. À l'ère de la patristique, ils ont occupé des sièges épiscopaux et ils ont fait de la théologie homilétique et catéchétique. Au Moyen Âge et par la suite, dans les chaires universitaires à l'intérieur d'une chrétienté bien établie, ils ont fait de la théologie savante et scolaire (scolastique). Au XIX siècle et jusqu'à Vatican II, les théologiens se retrouvent surtout dans les séminaires et font une «théologie de manuel» qui vise la formation des futurs prêtres (voir Moingt 1977).

Qu'en est-il de nos jours? Il y a certes une diversité de lieux de théologie, mais la plupart des théologiens les cherchent. Quelques-uns travaillent dans les antichambres des lieux magistériels où s'exercent surtout des tâches de vigilance. Quelques théologiens et théologiennes émigrent vers d'autres lieux universitaires, emportant avec eux les dépouilles de leur ancien discours théologique. Le plus grand nombre gardent leur poste d'enseignant à l'université, mais ils s'adressent à des auditoires nouveaux, de moins en moins nombreux et de plus en plus variés. Depuis les années 1965, nous sommes passés d'un auditoire de séminaristes et de religieux à celui des religieuses et des laïcs engagés en catéchèse et en pastorale. Depuis quelques années, les séminaristes se comptent sur les doigts d'une seule main et même les laïcs, qui se préparent à des tâches pastorales, sont moins nombreux d'année en année. La plupart des jeunes étudiants et étudiantes qui fréquentent les cours de théologie n'ont pas l'intention d'obtenir un grade en 
théologie, mais plutôt de compléter leur programme académique dans un domaine qui les intéresse et qui pique leur curiosité. À ces étudiants et étudiantes qui se montrent intéressés et sérieux, mais qui sont éloignés de la culture et de la pratique chrétiennes, enseignons-nous encore la théologie? Une solide catéchèse, tout au plus! Aux études supérieures, où les étudiants étrangers deviennent plus nombreux, il y a quelques étudiants d'ici qui se lancent dans des recherches doctorales, même si leur avenir professionnel n'est pas des plus prometteur.

Tout en tenant compte des contributions occasionnelles aux communautés chrétiennes, il m'apparaît que le lieu de la théologie est de moins en moins l'Église et qu'ainsi le théologien prend ses distances de la foi vécue dans les communautés chrétiennes. C'est pourquoi il est nécessaire de nous demander si la disparité et la dissémination des lieux où se fait la théologie vont renouveler et enrichir le discours théologique ou, au contraire, achever de le désagréger. La question n'est plus, dans un sens: "D'où parle le théologien? ", mais plutôt : "Y aura-t-il des lieux où on l'écoutera ? "Et puisque nous parlons du lieu de production du discours théologique, il ne faut pas sous-estimer la "version économique» de la même question. Pour assurer sa subsistance, le théologien doit s'adresser à des groupes d'étudiants et d'étudiantes assez nombreux qui justifieront son poste auprès des administrateurs de l'université. Cette donnée concrète entraînera dans les prochaines années des conséquences qui trancheront le procès de la place de la théologie dans le milieu universitaire. Plusieurs théologiens ne sont pas à l'aise dans ces considérations d'ordre économique qui semblent offenser la noblesse de la théologie, mais ils doivent finalement admettre qu'ils ont besoin d'un salaire pour vivre et que le budget de la bibliothèque universitaire s'établit habituellement selon le nombre d'étudiants et d'étudiantes.

On ne cesse de répéter que les gens, même s'ils ont pris leur distance de l'Église, sont épris de spiritualité plus que jamais et qu'ils cherchent des réponses aux grandes questions de l'existence. Le discours religieux trouve encore des preneurs, cependant, ce n'est pas nécessairement le discours des théologiens qui se vend le mieux, mais celui de ses concurrents, même si la théologie offre de la bonne marchandise. Les contemporains sont devenus soupçonneux à l'égard des théologiens qui, en plus d'être liés à une Église qui leur semble toujours autoritaire et même proche de l'intolérance, fondent leur discours sur l'autorité d'une révélation et de textes d'un passé lointain. C'est pourquoi la théologie subit le même sort que l'Église d'ici: elle devient de plus en plus une étrangère qui doit se faire discrète dans 
l'université et la société. La théologie court le risque de ne plus être reconnue comme une discipline académique parce qu'elle donne l'impression de contredire la liberté de pensée. Elle est de plus en plus mal à l'aise dans l'université, parce que sa place ne peut se séparer de celle que la société et l'État assignent à l'Église. La théologie est-elle donc condamnée à l'exil ?

\section{Les tentations du théologien}

Devant la clientèle devenue une denrée rare et devant la concurrence, qu'estce que le théologien peut faire, surtout à un moment où la théologie universitaire se doit d'être discrète, puisqu'elle est une discipline qui, du moins à première vue, contredit la mission de neutralité religieuse de l'université ? La place de la théologie ne saurait être autre que celle qui est reconnue à l'Église dans nos sociétés sécularisées et laïques. En effet, comment admettre à l'intérieur de l'université une discipline qui, de sa nature même, ne jouit pas de la liberté des autres disciplines en raison de sa soumission aux autorités de l'Église? On ne saurait cacher le malaise et le dilemme du théologien dans le milieu universitaire où est crédible celui qui pense par lui-même. N'est-il pas théologien par sa fidélité et son allégeance à l'Église et à son magistère?

Une double tentation guette le théologien. Tout d'abord, il peut être tenté de se réfugier dans un lieu nettement ecclésial, soit dans un séminaire, dans une école de la foi, soit dans un service ou un organisme de l'Église. Nous remarquons que des théologiens, en divers pays, prennent ce chemin et qu'ils ont le soutien et l'encouragement des autorités ecclésiales. Dans ce contexte, la théologie revêt une tonalité particulière et orientée. Elle risque de s'éloigner de la culture contemporaine et surtout de ne plus chercher à rendre la foi chrétienne "intelligible " pour ceux et celles qui sont en quête de sens dans une culture sécularisée. La ferveur ne remplacera jamais l'intelligence et la révélation chrétienne a toujours besoin de la rationalité et de concepts pour rejoindre les hommes et les femmes appelés à l'accueillir.

La seconde tentation est de transformer la théologie en science de la religion. Pour être reconnue à l'université, pour résoudre son complexe d'infériorité et aussi pour recruter une nouvelle clientèle dans le contexte social et religieux d'aujourd'hui, le théologien est tenté d'utiliser les approches des sciences de la religion en les adaptant à son savoir. La théologie court ainsi le risque de perdre son propre lieu de production, qui est la foi de la communauté chrétienne, et de s'égarer dans un lieu où le «religieux » devient un objet neutre de science. Quant à la foi chrétienne, elle se 
transforme en objet d'étude, un vis-à-vis neutre sans implication personnelle, afin de mieux assurer un regard objectif et scientifique. Permettez-moi d'évoquer ici le modernisme du début du $\mathrm{XX}^{\mathrm{e}}$ siècle qui illustre la rencontre de la foi chrétienne avec les sciences religieuses naissantes, notamment l'histoire. Alfred Loisy (1857-1940), dans L'Évangile et l'Église (1902) et Autour d'un petit livre (1903), nous présente un essai ou une tentative de réconciliation de la foi chrétienne avec la pensée moderne devenue plus rationnelle, autonome et familière avec les méthodes historico-critiques. Son projet ne manquait pas d'actualité et de pertinence, mais il aboutit à réduire le christianisme à une vague religion de l'humanité et à un humanisme d'inspiration chrétienne (voir Provencher 1983; 1995). Il faut admettre que le climat de suspicion et d'oppression, dans l'Église de ce temps, ne favorisait pas la réalisation du projet de Loisy. Tout au long du $\mathrm{XX}^{\mathrm{e}}$ siècle, où la foi chrétienne a été aux prises avec la modernité, le théologien s'est fait historien, sociologue, psychanalyste, anthropologue, marxiste, philosophe du langage. Il est certain que la théologie a beaucoup à recevoir des diverses sciences humaines et, de fait, elle en a profité grandement en devenant plus attentive à la richesse et à la complexité de l'être humain et de son insertion dans la modernité. De même que la théologie au Moyen Âge s'était façonnée en puisant chez Aristote, de même la théologie contemporaine apporte une intelligence renouvelée de la foi chrétienne grâce aux acquis des philosophies et des sciences humaines.

Cette alliance avec les sciences de la religion et les autres disciplines n'est pas sans soulever de nouvelles questions et même des malaises inquiétants pour l'avenir de la théologie. Cherchant un peu partout et empruntant de tous côtés ce qu'il peut bien avoir à dire, le théologien en vient à transformer son savoir en science de la religion. Ne prépare-t-il pas ainsi «sa propre oraison funèbre " ? En d'autres mots, en empruntant d'autres lieux que le sien, la théologie n'est-elle pas en train de perdre son propre lieu? Suis-je trop influencé par Michel de Certeau qui n'était pas tendre à l'égard des sciences religieuses du fait de leur "mollesse épistémologique », les considérant comme des «mixtes » qui ont tout simplement une fonction de transition entre les théologies d'hier et les sciences d'aujourd'hui. Certeau n'était pas plus tendre pour la théologie "qui prétend domestiquer les sciences humaines ": "Cette théologie meurt du dedans, avant même que son nom disparaisse ${ }^{3} . »(1987,247)$ Au XIX ${ }^{\mathrm{e}}$ siècle, le cardinal Newman avait déjà

3. Sur la position de cet auteur, voir Geffré 1991. 
perçu ce danger qui guette la théologie: "Si la théologie n'était pas là pour défendre ses frontières et empêcher les empiétements, [...] si la théologie est empêchée d'occuper son propre territoire, des sciences tout à fait étrangères à la théologie, ne manqueront pas d'en prendre possession.» (Newman 1945, 385) Dans nos milieux, on ne semble pas détecter cette menace de l'éviction de la théologie. Cette situation est inquiétante à la fois pour l'Église et la société.

Il ne faudrait pas conclure à l'impertinence et à l'insignifiance de la théologie. Michel de Certeau a toujours cherché, en marge de la théologie professionnelle, à rendre "pensable » le christianisme dans une société qui n'est plus religieuse. Avec une lucidité décapante, ce penseur nous aide à prendre conscience du non-lieu de la théologie dans la société moderne et sécularisée. Il s'agirait alors plus que d'un simple et momentané "déplacement ", mais d'une rupture inédite qui nous contraint à faire le deuil d'un certain nombre de certitudes confortables et à nous mettre en route vers un lieu non encore tracé clairement.

\section{La grâce de l'exil}

Même si nous sommes contraints à faire le deuil d'une certaine pratique de la théologie et à constater son non-lieu dans le champ du savoir contemporain, il ne convient pas de conclure à la mort de la théologie. S'agiraitil, selon l'expression du mystique et controversé Surin, d'un «heureux naufrage " ? Michel de Certeau nous fait comprendre que les ruptures peuvent être «instauratrices de sens". Ne pouvons-nous pas considérer que le tombeau vide et l'absence du corps du Crucifié du Golgotha sont les conditions du jaillissement de la foi, de l'avènement du corps de l'Église et du corps des Écritures ? En conséquence, il est normal que la théologie soit en rupture, mieux en exode et en exil ${ }^{4}$. Évitons cependant de faire un transfert hâtif de l'exil du peuple juif sur la situation actuelle de la théologie. Il reste que cette référence biblique nous remet en question et nous éclaire au cœur de nos inquiétudes et de nos tâtonnements. Cette expérience de nos ancêtres dans la foi rencontre certainement un écho dans la théologie de chez nous. La théologie en marge rejoint dans un sens l'ancien Israël en exil et dans la diaspora. C'est sur les bords des fleuves de Babylone qu'Israël prit une conscience plus vive de son identité de peuple choisi et redécouvrit

4. Joseph Moingt qualifie la théologie de Michel de Certeau de «théologie en exil» (voir Moingt 1991). 
le cœur de sa foi au Dieu unique, son Libérateur et son Créateur. De même, dans une terre étrangère, la théologie d'aujourd'hui a l'occasion de se dépouiller de ses lourdeurs et de devenir plus libre, afin d'écouter à nouveau la Parole faite chair jadis, mais toujours vivante et actuelle. Michel de Certeau nous rappelle que la théologie est toujours l'écho d'une parole unique, venue d'ailleurs, mais fragile: "Comme les Juifs privés de pays, sans lieu propre et donc sans histoire (il y a histoire là où il y a un lieu), après la destruction du Temple, les croyants sont livrés à la route avec des textes comme bagages. » $(1987,303)$

La théologie d'aujourd'hui n'a plus de terre sainte, ni de temple; elle est libre et peut habiter tous les lieux où on cherche le sens de l'existence, qu'il s'agisse de la philosophie, des sciences humaines, de l'éthique. C'est certes une situation inconfortable et génératrice de multiples tensions. Mais en dépit même de ce qui lui manque, elle ne doit pas renoncer à introduire une exigence critique à l'égard des discours culturels, religieux ou politiques, qui seraient tentés de se fermer sur eux-mêmes. En effet, la théologie a toujours la mission de faire entendre la "différence " chrétienne, cette différence qui rejoint toute quête de sens comme quête d'un «Autre» tellement discret qu'il paraît absent. Cette "différence» que la théologie a la mission de faire entendre est finalement celle de la foi chrétienne. S'inspirant de Franz Rosenberg, Jean-Claude Guillebaud exprime le rôle de la foi dans la quête du sens en ces termes: «La foi est précisément ce qui reste, la trace indélébile, indicible, mais qui demeure contre vents et marées, attestant du sens. Le reste est la parcelle qui demeure intacte en dépit de tout; la lueur qui témoigne sans imposer. Ce reste fait fond sur la seule puissance du message. » $(2003,321)$

Ainsi, la théologie ne serait pas seulement en crise, fatiguée et même en phase terminale, à la façon de l'Église où elle trouve son lieu. Dans la situation présente, elle a l'occasion, mieux la grâce, de sortir de ses enclos sacrés et de s'aventurer sur des chemins nouveaux et inédits avec plus d'élan, parce qu'elle est moins lourde. Elle peut prendre son second souffle, à la condition toutefois de se concentrer sur l'essentiel du mystère chrétien. Et la théologie a la mission de déployer la richesse du mystère chrétien et d'en faire ressortir sa lumière dans la société d'aujourd'hui, et sur plus d'un aspect elle est encore la seule à pouvoir le faire. Pour réaliser sa mission, elle doit quitter les champs clos du religieux et inventer un autre langage de la foi, en solidarité avec les autres, croyants et incroyants, avec leurs souffrances, leurs soucis, leurs projets. Cette théologie apportera des réponses, 
sans prétention toutefois, à de vraies questions des gens d'aujourd'hui, à leur quête du sens et à leur recherche de Dieu. Elle ne peut réaliser ses objectifs quand elle se limite à élaborer des discours qui s'enracinent dans des milieux fermés, même s'ils sont universitaires, ou encore de l'idéologie religieuse.

Dans le contexte actuel de déconfessionnalisation des écoles et de neutralité de l'État, il est grand temps de nous demander si l'existence des facultés de théologie n'est pas menacée. La théologie doit s'élaborer en liens étroits avec la communauté chrétienne qui est nécessairement d'une confession particulière. Puisque l'État prend de moins en moins le risque de privilégier une religion et une confession chrétiennes au détriment des autres, les facultés de théologie ne pourront presque plus assurer leurs liens avec l'Église catholique dont l'influence devient de plus en plus marginale. Il est donc urgent que les dirigeants de l'Église encouragent la création de nouveaux «lieux» d'enseignement et de recherche, autres que les séminaires, pour que la théologie puisse continuer à jouer son rôle de transmission et d'actualisation de l'héritage chrétien dans la société contemporaine.

\section{Conclusion}

L'Église est devenue une étrangère dans notre société sécularisée et postchrétienne. De plus en plus, on s'organise et on vit sans elle. On l'ignore ou on la juge démodée et rétrograde. Son message n'interpelle presque plus sur des questions vitales et concrètes de l'existence humaine. Le meilleur de sa parole, on le retrouve dans les valeurs promues par la société. Marcel Gauchet reconnaît qu'elle a joué son rôle et donné le meilleur d'elle-même et qu'elle peut donc se retirer avec le sentiment du devoir accompli. En conséquence, la théologie devient de plus en plus isolée et discrète, comme l'Église qui est son lieu vital. L'exil de l'Église dans la société d'ici n'est pas sans répercussion sur la théologie.

Mais si l'Église était en train de renaître ailleurs et de s'engager dans des chemins nouveaux, à peine tracés, là où les gens cherchent un sens à leur vie et à l'histoire, là où les gens souffrent, là où ils s'entraident, la théologie à son tour serait en train de renaître, d'occuper une place originale en étant toujours la foi qui cherche à être intelligible. L'exil est une situation pénible qui ressemble souvent à la phase terminale de toute personne, mais elle est aussi une grâce qui mène à ce lieu où l'Évangile résonne et annonce toujours du nouveau qu'il crée tout en le révélant. La fin du monde n'est-elle 
pas la toile de fond du Nouveau Testament, mais aussi le recommencement et la nouveauté promise. La théologie, qui assume et profite de sa situation de précarité, d'exil et de présence dans ce qui est encore trop souvent marginalisé, peut entrevoir l'aurore d'un nouveau matin.

L'avenir de l'Église et de la théologie est déjà commencé. Il se prépare discrètement et parfois, il faut en convenir, loin des décisions et des orientations officielles. Il est possible que nous vivions un retour d'exil, mais non un retour à la théologie que nous avons étudiée et enseignée. Que sera la théologie dans ces lieux qui sont apparemment dans les marges? Nous ne pouvons pas prévoir son avenir qui «ne sera pas qu'un aujourd'hui agrandi et meilleur» (Gauchet 1985, 266), mais nous pouvons l'inventer en étant fidèles aux appels nouveaux de l'Esprit et de la société et en acceptant de faire de la théologie dans les terres encore en friche, là où l'humanité nouvelle et l'Église de demain sont en train de germer.

\section{Références}

Bellet, M. (2001), La quatrième hypothèse. Sur l'avenir du christianisme, Paris, Desclée de Brouwer.

Bousquet, F., H.-J. Cagey, G. Médevielle et J.-L. Souletie, dir. (2002), La responsabilité des théologiens. Mélanges offerts à Joseph Doré, Paris, Desclée de Brouwer.

Certeau, M. de (1987), La faiblesse de croire / texte établi et présenté par L. Giard, Paris, Seuil.

DuQuoc, C. (2002), La théologie en exil. Le défi de sa survie dans la culture contemporaine, Paris, Bayard.

Gauchet, M. (1985), Le désenchantement du monde. Une histoire politique de la religion, Paris, Gallimard.

Geffré, C. (1991), "Le non-lieu de la théologie chez Michel de Certeau », dans C. Geffré, dir., Michel de Certeau ou la différence chrétienne, Paris, Cerf, p. 159-180.

Guillebaud, J.-C. (2003), Le gô̂t de l'avenir, Paris, Seuil.

Jacquard, A. (2003), Dieu?, Paris, Stock / Bayard.

Lafont, G. (2003), Promenade en théologie, Paris, Lethielleux.

Moingt, J. (1977), «Perte du lieu, rapt du lieu », dans Le déplacement de la théologie, Paris, Beauchesne (Le Point théologique 21), p. 151-156. 
